\section{Obtención y caracterización de astaxantina de la microalga Haematococcus pluvialis}

\section{Preparation and characterization of astaxanthin from microalgae Haematococcus pluvialis}

\author{
Nancy M. Córdoba-Castro*, Nadine L. Acero-Reyes**, Luisa F. Duque-Buitrago**, L. Juliana \\ Jiménez-Aguilar** \& Johanna Andrea Serna-Jiménez**
}

*Facultad de Ingeniería, Programa de Ingeniería de Procesos, Universidad Mariana, Pasto Nariño **Facultad de Ingeniería, Programa de Ingeniería Agroindustrial, Universidad La Gran Colombia Seccional Armenia.

\section{Resumen}

La Astaxantina es un carotinoide perteneciente a la familia de las xantofilas por la presencia de oxígeno en su estructura química. Su uso se ha difundido en la industria farmacéutica y pecuaria, siendo la primera la que más potencial presenta debido a su uso como antioxidante. Existen diferentes fuentes para la obtención de astaxantina, sin embargo; en los últimos años ha tomado gran relevancia la obtención de esta biomolécula a partir de microorganismos fotosintéticos como las microalgas, las cuales pueden producir diferentes metabolitos aprovechando la energía solar y capturando el CO2 de la atmósfera. Se ha encontrado que la microalga Haematococcus pluvialis es una de las microalgas que mayor potencial presenta en la producción de astaxantina. Esta producción se realiza bajo condiciones de estrés por represión de nutrientes, intensidades de luz, entre otros. Debido a la importancia que reviste la astaxantina y su aprovechamiento a nivel industrial se han evaluado diferentes métodos para su extracción y cuantificación tales como: extracción con solvente, con fluidos supercríticos, cuantificación por HPLC con fase reversa acoplada a espectrofotometría de masas, siendo estos los más desarrollados para este tipo biomolécula, la cual se ha cuantificado en el espectro UV/Visible por las características propias de la molécula, como son sus enlaces conjugados. Sin embargo, al tratarse de un carotinoide cada una de las metodologías de extracción y cuantificación deben ser evaluadas cuidadosamente con el fin de que esta no sea degradada. El presente artículo presenta una breve descripción de los métodos más usado en la extracción y cuantificación de la astaxantina de $\mathrm{H}$. pluvialis y las perspectivas de uso de esta biomolécula

Palabras clave: Astaxantina, Haematococcus, HPLC, carotenoide

\begin{abstract}
Astaxanthin is a carotenoid belonging to the family of xanthophylls by the presence of oxygen in their chemical structure. Use is widespread in the pharmaceutical industry and pisciculture, with the first presents the greatest potential because of its use as an antioxidant. There are different sources for obtaining astaxanthin, however; in recent years has become very important to obtain this biomolecule from photosynthetic microorganisms such as microalgae, which can produce different metabolites using solar energy and capturing $\mathrm{CO} 2$ from the atmosphere. It has found that the microalga Haematococcus pluvialis is one of the microalgae has the greatest potential in the production of astaxanthin. This production is performed under conditions of stress by suppressing nutrients, light intensity, and others. Because of the importance which is of astaxanthin and its use at industrial level have been evaluated different methods for extraction and quantification of such as solvent extraction, supercritical fluid, quantification by reverse phase HPLC coupled with mass spectrometry, with the most often developed for this type biomolecule, which has quantified in the UV / Visible by the characteristics of the molecule, as are their conjugated bonds. However, being a carotenoid each of the methods of extraction and quantification must be carefully evaluated in order that it not degraded. This article presents a brief description of the methods used in the extraction and quantification of astaxanthin $\mathrm{H}$. pluvialis and prospects for use of this biomolecule.
\end{abstract}

Keywords: Astaxanthin, Haematococcus, HPLC, carotinoide
Recibido: 18/02/2015

Revisado: 05/07/2015

Aceptado: 10/12/2015

Correspondencia de autor: andreasernajimenez@hotmail. com

(C) 2015 Universidad La Gran Colombia. Este es un artículo de acceso abierto, distribuido bajo los términos de la licencia Creative Commons Attribution License, que permite el uso ilimitado, distribución y reproducción en cualquier medio, siempre que el autor original y la fuente se acrediten.

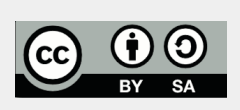

\section{Cómo citar:}

Córdoba-Castro, NM., AceroReyes, N.L., Duque-Buitrago, L.F., Jiménez-Aguilar, L.J., Serna-Jiménez， J.A. (2015) Obtención y caracterización de astaxantina de la microalga Haematococcus Pluvialis. UGCiencia 21, 73-82. 


\section{Introducción}

Las microalgas son organismos fotosintéticos de elevado valor biotecnológico debido a que pueden presentar gran variedad de aplicaciones como la capacidad de producir biodiesel (Fernández-Linares, et al., 2012), propiedades biorremediantes en aguas residuales (Matamoros, et al., 2016) o ser útiles en la producción de bioproteína para la alimentación animal y humana (Kim, 2015; kiron, et al. 2012), así como también, poseen la característica especial de acumular metabolitos de interés como los ácidos grasos poli-insaturados de cadena larga y los carotenoides (Ramírez, 2013; Orosa, et al., 2005)

H. pluvialis es una microalga del orden Volvocales y familia Haematococcaceae, su extendido estudio biotecnológico se debe a su capacidad, de producir dentro de su metabolismo, proteína y astaxantina; este es un compuesto de la familia de los carotenoides responsable de la coloración rojiza de algunos crustáceos, mariscos y de la carne de salmónidos, siendo las microalgas la mayor fuente de astaxantina natural conocida (Algatechnologies, 2015; Gómez, et al., 2013). La H. pluvialis puede llegar acumular hasta 5,02\% de astaxantina del peso en célula seca y conteniendo $36,7 \mathrm{mg} / \mathrm{g}$ de trans-astaxantina $-73,1 \%$ y $13,5 \mathrm{mg} / \mathrm{g}$ de cis-astaxantinas $-26,9 \%$-; en forma de monoésteres $(80 \%)$ y diésteres $(15 \%)$ y (5\%) libre (Roldán, 2012).

La presencia de oxígeno en forma de grupos hidroxilos $(\mathrm{OH})$ y carbonilos $(\mathrm{C}=\mathrm{O})$ en la estructura química de la astaxantina; le confiere algunas propiedades diferenciadoras como su capacidad de esterificación, su naturaleza polar (Seabra y Pedrosa, 2010) y su alta capacidad antioxidante que presentada diversos efectos beneficiosos en enfermedades relacionadas con el daño oxidativo (Xuebo y Toshihiko, 2007). La capacidad de esterificación con diferentes ácidos grasos depende del origen de esta, aunque también puede encontrarse en forma libre con grupos hidroxilos no esterificados. Algunos carotenoides apolares como el licopeno y $\beta$-caroteno muestran efectos pro-oxidativos mientras que la astaxantina puede reducir la peroxidación lipídica hasta en un $40 \%$ (Seabra y Pedrosa, 2010) aunque esa polaridad característica interfiere con una fácil extracción desde la matriz celular, donde es insoluble en agua, parcialmente soluble en etanol y completamente soluble en acetona y n-hexano (Harker, Tsavalos Young, 1996)

La producción de este metabolito secundario está fuertemente relacionada con los cambios morfológicos producidos en la microalga desde un estado vegetativo verde en el que se da el crecimiento, hasta un estado rojo de alta acumulación de astaxantina. Estos cambios pueden ser inducidos por diversos factores como la deficiencia de nutrientes, la alta intensidad lumínica, la salinidad del medio y las altas temperaturas, entre otros (Harker, Tsavalos, y Young, 1996; Wan, et al., 2014) (Vidhyavathi, et al., 2007). El color generado por la astaxantina protege los quistes maduros del detrimento por los efectos de la radiación UV cuando estos están expuestos a los rayos solares directos
(Camacho, González y Klotz, 2013; Suyono, et al., 2015; Harker, Tsavalos y Young, 1996)

La importancia de la astaxantina no solo está relacionada con su capacidad colorante, sino también por su poder antioxidante que ha mostrado ser mayor que R-caroteno, alfa-caroteno, betacaroteno y luteína. En la inhibición iniciada por radicales, ya sea, por medio del quelato de hierro (Fe3+/ADP) y un componente reductor (NADP) o por medio de la peroxidación lipídica en hígado de rata por el iniciador hidrosoluble azo 2,2'-azobis (2-amidinopropano), la astaxantina es tan eficaz como R-tocoferol (Naguib, 2000), eliminando los radicales libres y especies reactivas de oxígeno e inhibiendo la peroxidación lipídica (Algatechnologies Ltd., 2015; Sila, y otros, 2015).

\section{Microorganismo}

H. pluvialis presenta un ciclo con diferentes formas celulares respondiendo a los estímulos del medio, en el tiempo se caracteriza por mostrar 3 morfologías: Como lo menciona Domínguez (2006) vegetativa (verde con presencia de flagelos), palmella (verde, esférica sin flagelos) y aplanóspora (roja, esférica sin flagelos) es en esta última donde se produce las astaxantina como respuesta al medio (Figura. 1)

Figura 1. Ciclo celular del alga. Fuente: (Dominguez A, 2006)

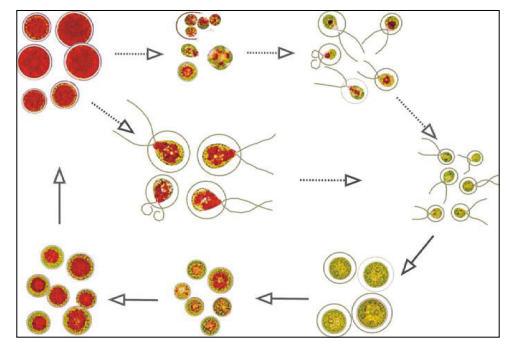

Es así como su ciclo depende del tipo de producción que se desee hacer, así mismo se estimula una alta producción de biomasa con estadío de palmella para que se dé la producción de la aplanóspora reflejándose en una alta cantidad de astaxantina, es de aclarar que la astaxantina es producida por la microalga de forma intracelular (no es excretada al medio) y este estadío depende exclusivamente de las condiciones de estrés a la que se exponga el microorganismo. Como lo menciona Domínguez (2006), La aplanóspora funciona entonces como una estructura de resistencia a las condiciones del medio, si el medio deja de ser "hostil" la microalga es capaz de revertir el proceso retornando a la configuración vegetativa convirtiéndose estas características en cíclicas.

Es así, como la microalga presenta dos necesidades de cultivo a condiciones diferentes para la producción de biomasa y para la producción de astaxantina. (Harker, Tsavalos, \& Young, 1996; Choi, et al., 2011; Ahmed, et al., 2015). 
En cuanto al contenido de carotenoides la empresa Cyanotech, líder en la producción de astaxantina reporta que en la microalga $H$. pluvialis; la fracción carotenoide contiene aproximadamente el $70 \%$ monoésteres de astaxantina, $10 \%$ de diésteres de astaxantina, un $5 \%$ de astaxantina libre, y el $15 \%$ restante se compone de una mezcla de $\beta$-caroteno, cantaxantina, luteína y otros carotenoides. (Wenduo, et al., 2014)

\section{Caracterización astaxantina}

La Astaxantina es un carotenoide perteneciente a la familia de las xantofilas por la presencia de oxígeno en su estructura. Las xantofilas se caracterizan por ser pigmentos y se han descubierto sus altas propiedades farmacéuticas. En el caso especial de la astaxantina se reconoce como un antioxidante de alta eficiencia. Su descripción se muestra en la tabla 1 .

Tabla 1. Características de la Astaxantina

\begin{tabular}{|c|c|}
\hline Característica & Descripción \\
\hline fuente & Haematococcus pluvialis \\
\hline Nombre químico & Astaxantina \\
\hline Peso molecular & 596,8 Daltons \\
\hline Formula química & $\mathrm{C}_{40} \mathrm{H}_{52} \mathrm{O}_{4}$ \\
\hline Estado físico & Aceite viscoso \\
\hline solubilidad & $\begin{array}{l}\text { Insoluble en agua o agua caliente, } \\
\text { parcialmente soluble en etanol. Soluble en } \\
\text { hexano, acetona éter. }\end{array}$ \\
\hline Color & Rojo oscuro \\
\hline almacenamiento & $\begin{array}{l}\text { Almacenar en bolsas oscuras selladas a } \\
10^{\circ} \mathrm{C} \text { sin exceder los } 69^{\circ} \mathrm{C} \text {. }\end{array}$ \\
\hline
\end{tabular}

El nombre químico de la astaxantina es 3, 3'-dihidroxi-b, b,caroteno-4, 4' diona (Guerin, Huntley, \& Olaizola, 2003). La astaxantina presenta 3 isómeros; $3 \mathrm{~S}, 3^{`} \mathrm{~S}$ y $3 \mathrm{R}, 3^{`} \mathrm{R}$ son enantiómeros con actividad óptica opuesta y $3 R, 3{ }^{`} R$ tiene actividad óptica meso (GRAS) (figura 2).

Figura 2. Isómeros de astaxantina. Fuente: Hagen, Siegmund y Breune, (2002)

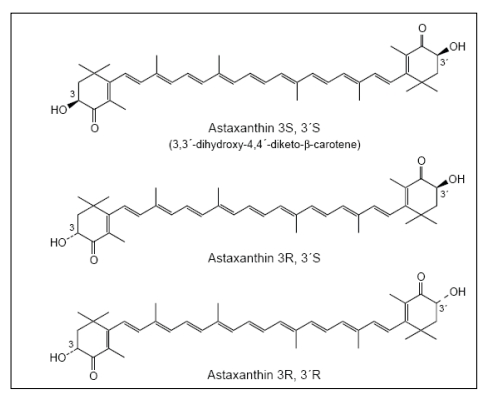

La estéreoespecifidad de las moléculas está directamente relacionada con su actividad biológica, por lo que, los carotenoides naturales son más biodisponibles y presentan mayor efectividad (BioNutrition, 2008). Por sus características estructurales y las posiciones 4 y 4' aumentan la capacidad antioxidante aún más que las reportadas por otros carotenos tiene la capacidad de capturar los radiales libres (Guerin, Huntley, \& Olaizola, 2003)

Por su característica hidrofóbica es común encontrarla en algunos animales y crustáceos ligada a las proteínas y lípidos presentes en la carne, por lo que en el momento de la cocción la molécula puede sufrir modificaciones y alteraciones biológicas (Sila, et al., 2015; Rao, et al., 2013; Anarjan, et al., 2012).

A diferencia de otros carotenoides esta no es precursor de vitamina A por lo que su consumo no genera vitaminosis.

\section{Técnicas de obtención}

Para extraer, cuantificar y estabilizar la astaxantina producida por la microalga $H$. pluvialis, teniendo en cuenta la aplicabilidad comercial se han investigado diferentes procesos.

Los carotenoides, debido a la alta conjugación de enlaces dobles presentes en sus moléculas se descomponen por efecto de la luz, la temperatura y el aire. La luz favorece reacciones fotoquímicas que cambian la estructura original del carotenoide (por ejemplo isomerismo cis y trans) es un factor a considerar al momento de realizar su extracción. El calor también favorece reacciones térmicas de degradación. El aire debido al oxígeno favorece la oxigenación de los enlaces dobles a funciones epóxido, hidroxilos y peróxidos, entre otros. Por estas razones la extracción de carotenoides se debe preferiblemente realizar en condiciones de ausencia de luz, a temperatura ambiente o menor, y en ausencia de oxígeno (por ejemplo con atmosferas modificadas) (Lababpour, et al., 2005). Además se debe realizar lo más rápido posible, y a partir de material biológico fresco, para evitar la degradación por la acción conjunta de estos factores adversos (Martínez, 2003; Tripathi, et al., 1999).

Debido a que los carotenoides en su mayoría son solubles en solventes apolares como éter etílico, benceno, cloroformo, acetona, acetato de etilo, entre otros; como lo menciona Martínez (2003), estos se deben extraer de material biológico fresco, el cual presenta un alto contenido de agua que dificulta una extracción eficiente, por esto es conveniente eliminar el agua. El tejido deshidratado se puede entonces extraer con un solvente apolar. Una alternativa a este proceso de deshidratación es la liofilización, la cual resulta ventajosa porque se realiza a baja temperatura y al vacío, eliminando la posibilidad de degradación por altas temperaturas y presencia de aire (Martínez, 2003).

Martínez (2003) en su trabajo en técnicas de obtención de carotenoides, menciona que las mezclas de carotenos y las xantofilas mono- y dihidroxiladas pueden separarse agitando una 
solución en éter de petróleo con un volumen de metanol al 90 $\%$; las xantofilas dihidroxiladas quedan en la fase metanólica, las monohidroxiladas y los carotenos quedan en la fase etérea. Repitiendo este proceso con la fase etérea se separan en la fase metanólica las xantofilas monohidroxiladas, y en la fase etérea quedan los carotenos. Las xantofilas separadas en las fases metanólicas pueden recuperarse extrayéndolas con éter etílico (Martínez, 2003).

Una vez obtenido el extracto o los extractos de carotenoides, estos se pueden separar y analizar por cromatografía en capa fina, en papel o en columna. Más recientemente y gracias al avance de los métodos cromatográficos instrumentales es posible el aislamiento rápido de carotenoides puros. En este sentido, La High Performance Liquid Cromatography (HPLC) es muy utilizada actualmente, debido a que se puede trabajar a bajas temperaturas, en ausencia de luz y aire, siendo una técnica muy sensible que permite la cuantificación y cualificación de los productos y caracterización de los diferentes carotenos presentes (Martínez, 2003).

\section{Procesos de extracción}

\section{Extracción con solvente}

En la técnica reportada por Miao, et al. (2006) se tomó una porción de muestra, utilizándose éter de petróleo para posterior centrifugación, finalmente el sobrenadante se llevó a recuperación utilizando un rotavapor para obtener finalmente el extracto; en este tipo de técnicas es importante cuidar la exposición al oxígeno así como las altas temperaturas que puedan llegar a degradar las muestras (Henriques, Silva, \& Rocha, 2007).

\section{Extracción con enzimas líticas}

Kobayashi y colaboradores, (1997) reportan la efectividad en la extracción de astaxantina de $H$. pluvialis utilizando una combinación de enzimas líticas. El procedimiento utilizado describe que primeramente las células fueron tratadas con el fin de extraer la clorofila con un tratamiento de acetona por 24 horas. Una vez las células libres de clorofila se liofilizaron y se trataron con enzimas líticas comerciales: Actinasa E, Celulasa, Quitinasa, Fangase I, Funcelase, Kitalasa, Novozym 23, pectinasa, proteasa, Proteinasa K, Tunicasa R70, Uskizyme, Yatalasa y Zymolyase. Las mezclas de reacción enzimática $(10 \mathrm{mg}$ de células, $10 \mathrm{mg}$ de cada enzima y $10 \mathrm{ml}$ de agua desionizada) se incubaron durante 24 horas a $37^{\circ} \mathrm{C}$. Las células tratadas con enzimas fueron recuperadas por centrifugación a $2000 \mathrm{~g}$ durante 10 minutos, y se lavó dos veces con agua.

La extracción de astaxantina se estimó como astaxantina libre y total, la primera se obtuvo después de 1hora de tratar las células con acetona al $90 \% \mathrm{v} / \mathrm{v}$ y la segunda después de la hora de tratamiento; se aplicó ultrasonido. La concentración de astaxantina se determinó espectrofotométricamente.
La kitalasa (principalmente la $\beta$-1, 3-glucanase preparada de Rhizoctonia solani), Celulasa (principalmete la $\beta$-1,4-glucanase preparada de Trichoderma sp.) presentaron una mayor extractibilidad de astaxantina (tabla 2). Por otra parte, cuando las células fueron pre-tratadas con acetona (libres de clorofila) se trataron con la combinación de las tres enzimas, la extractabilidad fue 3 veces más alta que con las células intactas.

Tabla 2. Efectos de enzimas líticas en extractabilidad de astaxantina a partir de $H$. pluvialis. Tomado de Kobayashi, et al., 1997

\begin{tabular}{|c|c|c|c|c|}
\hline \multicolumn{3}{|c|}{ Treatment with cell lytic enzymesa } & & \\
\hline \multirow[t]{2}{*}{ Kitalase } & \multirow[t]{2}{*}{ Cellulase } & \multirow{2}{*}{$\begin{array}{c}\text { Abalone } \\
\text { acetone powder }\end{array}$} & \multicolumn{2}{|c|}{$\begin{array}{l}\text { Extractability of } \\
\text { astaxanthin (\%) }\end{array}$} \\
\hline & & & Intact cells & Heated cells $s^{b}$ \\
\hline - & - & - & 0 & 14 \\
\hline+ & - & - & 8 & 32 \\
\hline - & + & - & 13 & 38 \\
\hline - & - & + & 1 & 18 \\
\hline+ & + & - & 16 & 50 \\
\hline+ & - & + & 17 & 48 \\
\hline - & + & + & 17 & 43 \\
\hline+ & + & + & 24 & 68 \\
\hline
\end{tabular}

Cuando las células intactas se liofilizaron en ausencia de acetona, la extractabilidad de astaxantina no aumentó, pero se incrementó ligeramente cuando hubo un calentamiento de las células (tabla 2). Sin embargo, cuando las células fueron tratadas con calor-acetona-tratamiento (libre de clorofila) y fueron liofilizadas, la extractabilidad fue significativamente mayor. Por lo tanto, la pared celular de las células resistentes requiere un pretatamiento de calor-acetona antes de la liofilización o reacción enzimática lítica.

La modificación de la pared de la célula antes de la extracción de pigmentos requiere tratamiento térmico-acetona (acompañada por el retiro de la clorofila) y liofilización posterior o tratamiento de enzimas líticas. Estos tratamientos combinados pueden ser útiles para el procesamiento de $H$. pluvialis y garantizar la conservación y extracción de astaxantina a partir de células encapsuladas (tabla 3).

Tabla 3. Efectos de la liofilización en extractabilidad de astaxantina a partir de células del H. pluvialis tomado de Kobayashi, et al., 1997

\begin{tabular}{cccc}
\hline $\begin{array}{c}\text { Acetone } \\
\text { concentration } \\
(\%)\end{array}$ & $\begin{array}{c}\text { Heating } \\
\text { of cells }\end{array}$ & $\begin{array}{c}\text { Lyophilization } \\
\text { of cells } s^{b}\end{array}$ & $\begin{array}{c}\text { Extractability of } \\
\text { astaxanthin (\%) }\end{array}$ \\
\hline 0 & - & - & 0 \\
0 & - & + & 0 \\
0 & + & - & 0 \\
0 & + & + & 9 \\
10 & + & + & 26 \\
20 & + & + & 50 \\
30 & + & + & 68 \\
40 & + & + & 60 \\
\hline
\end{tabular}




\section{Extracción de astaxantina por Soxhlet}

En investigación realizada por Thana, et al., (2008) se realizó la extracción de astaxantina en la microalga $H$. pluvialis, utilizando un Soxhlet, con el que se extrajo de una muestra de utilizando como solvente acetona, durante 6 horas hasta que el color del solvente condensado en el superior del aparato estaba claro (Thana, et al., 2008). Los rendimientos obtenidos fueron de $13,73 \mathrm{mg}$ o $2,75 \%$ del peso seco.

\section{Extracción con fluidos supercríticos}

La extracción con fluidos supercríticos (SFE) es una tecnología moderna con aplicaciones cada vez mayores en la industria de trasformación farmacéutica y alimentaria. El principio del proceso consiste en utilizar un fluido en condiciones supercríticas (presión-temperatura) en las que sus propiedades fisicoquímicas se encuentran entre las de un líquido y un gas (figura 3 ).

Figura 3. Diagrama de fase para el dióxido de carbono, tomado de Mendes, et al., (2003).

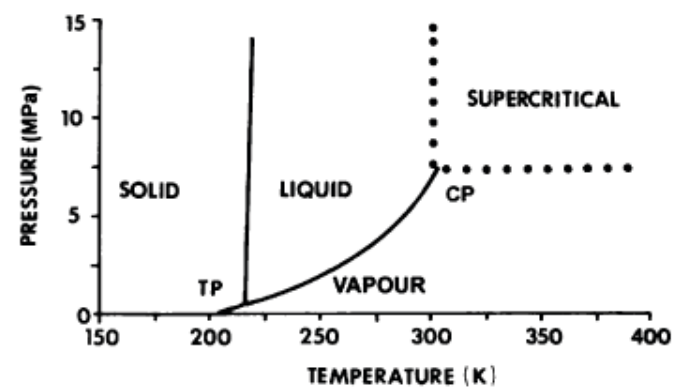

La temperatura y la presión están por encima los valores críticos; llevar el fluido a que posea propiedades especiales como la difusividad alta, de baja viscosidad y baja tensión superficial. Esto permite que el fluido a una mejor difusión a través naturales matriz sólida, y por lo tanto es mejor extraer los compuestos naturales en comparación con los disolventes líquidos convencionales. Cuando la presión y la temperatura se reducen, la pérdida de estas características especiales se produce. Por lo tanto, el soluto se puede extraer con el disolvente a condición supercrítica, y se separan cuando la presión y la temperatura se reducen por debajo de su estado crítico. (Jaime, et al., 2010) El solvente supercrítico más frecuentemente empleado en los alimentos y en la elaboración de productos naturales es el dióxido de carbono $\left(\mathrm{CO}_{2}\right)$, debido a su baja toxicidad y baja temperatura crítica. Los ajustes realizados en el estudio realizado por Thana, et al., (2008) lograron obtener en condiciones óptimas de $70^{\circ} \mathrm{C}$, $50 \mathrm{MPa}$, y 4 h $23.04 \mathrm{mg} / \mathrm{g}$ (2.3\%peso) de astaxantina de $H$. pluvialis.

\section{Caracterización espectral}

\section{Espectro UV}

Los pigmentos pueden absorber la luz específicamente en la región ultravioleta (UV) y el espectro visible, el resto se trasmite o refleja. La estructura responsable de la absorción de la luz es la agrupación cromóforo, que en carotenoides se caracteriza por dobles enlaces conjugados. Cada carotenoide se caracteriza por un espectro de absorción electrónica. Por lo tanto, la espectroscopia de absorción es una técnica importante en el análisis de carotenoides. (Valdugai, et al., 2009; You, Chul, \& Yeoung, 2005)

Por la estructura química de la astaxantina y la presencia de enlaces conjugados se puede identificar y cuantificar con la técnica espectrofotométrica. Este carotenoide absorbe entre 250 y $550 \mathrm{~nm}$, presentando un pico máximo en 488nm (figura 4) (Japanese Conference on the Biochemistry of Lipids, 2008).

Figura 4. Espectro UV de astaxantina en etanol. Fuente: (Japanese Conference on the Biochemistry of Lipids, 2008)

\section{Espectro infrarrojo}

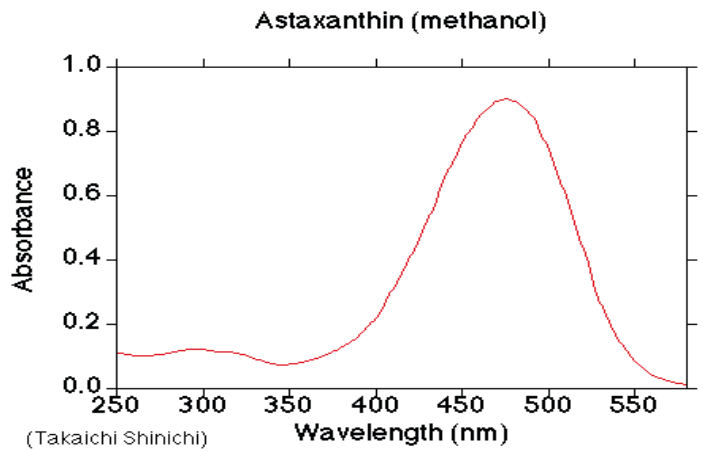

En el espectro infrarrojo la astaxantina presenta señales que muestra cada tipo de enlace característico de su estructura (figura 5).

Figura 5. Espectro infrarojo. Fuente: (Japanese Conference on the Biochemistry of Lipids, 2008)

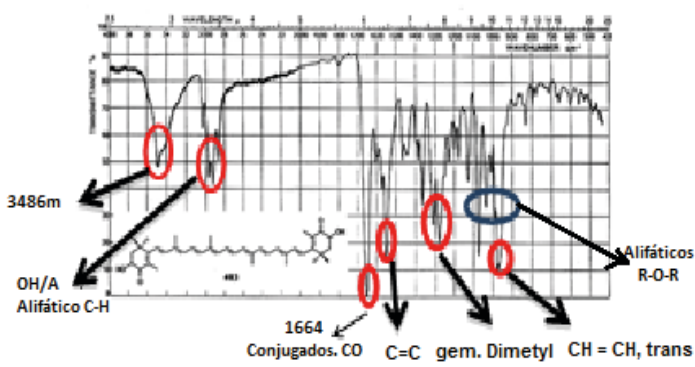




\section{Cromatografía}

Debido a la presencia de cadenas polienicas conjugadas en su molécula, los carotenoides son por lo general inestables en presencia de luz, calor, u oxígeno. El aislamiento, identificación y cuantificación de estos se dificulta ya que muchos otros compuestos pueden interferir en su aislamiento. El uso de la cromatografía de gases (CG) y espectrometría de GC-masas (GC-MS) no es adecuado porque los carotenoides son termolábiles. Solo el método de cromatografía líquida de alta eficiencia (HPLC) resulta útil, ya sea con detección UV/visible (UV/Vis), o mucho más conveniente con detección por espectrometría de masas (LC-MS).

HPLC de fase inversa (RP-HPLC) es el método preferido en el cual se usa con frecuencia una columna $\mathrm{C} 18$, frecuentemente con elución en gradiente (Sila, et al., 2015; Su, et al., 2014; Orosa, et al., 2005). Los detectores de absorbancia UV/ VIS carecen de la selectividad y la posibilidad de identificar cada especie molecular está limitada debido a que carotenoides individuales son estructuralmente muy similares. A diferencia de otras técnicas de ionización, xantofilas y carotenos forman tanto iones moleculares como moléculas protonadas en presencia de iones positivos APCI (ionización química a presión atmosférica (Rezanka, Nedbalova, \& Sigler, 2008). Debido a la naturaleza compleja de los carotenoides en el extracto celular de algas, es difícil separar e identificar cada pigmento dentro de ella. Yuan y sus colaboradores desarrollaron una técnica de cromatografía líquida (HPLC) método para separar e identificar los ésteres de astaxantina en Haematococcus lacustris encontrando que 5 monoésteres de astaxantina, representan el $79 \%$ de los carotenoides totales y son las principales formas de ésteres de astaxantina. Más tarde, el mismo grupo aislo y determinó los ésteres de astaxantina en $H$. pluvialis por el método HPLC (Miao, et al., 2006; Sila, et al., 2015; Su, et al., 2014; Orosa, Franqueira, Cid, \& Abalde, 2005)

Los datos de HPLC de cromatografía en la figura 2 demostraron diferentes tiempos de retención para cada componente en el extracto de $H$. pluvialis los carotenoides libres fueron eluidos entre 6 y 15 minutos, mientras que monoésteres astaxantina generalmente tenía de 20 a $34 \mathrm{~min}$, los diésteres se eluyeron después de 45 minutos. Según lo detectado por HPLC-DAD, la mayoría de los compuestos tenían una absorción máxima de aproximadamente $480 \mathrm{~nm}$, que es una absorción característica del esqueleto de astaxantina. Además, fue observado un pico de absorbancia a $400 \mathrm{~nm}$ (tiempo de retención de 35-40 minutos). Esto puede representar un conjunto de compuestos eluidos entre monoésteres y diésteres de astaxantina. HPLC se realizó en un Agilent 1100 con un detector de diodos (DAD), con una columna de fase reversa $\mathrm{C} 18$. La fase móvil consistió en $60 \mathrm{~min}$ de 83: 17 a 92:2 (acetona: agua). La tasa de flujo fue $0.8 \mathrm{~mL}$ min. (Miao, et al., 2006). En experimentos posteriores, un espectro de masas individuales correspondientes a la cima con la absorción a $480 \mathrm{~nm}$ fue producido por la MS. (figura 6, Tabla 4)
Figura 6. Cromatograma HPLC (DAD, $476 \mathrm{~nm}$ ) ingredientes en los extractos de $H$. pluvialis. Tomado de Miao, Lu, Li, \& Zeng (2006).

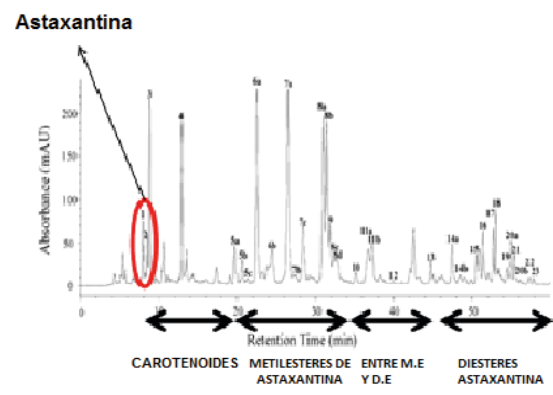

\section{Espectroscopia de masas}

En la figura 7 se muestra un ejemplo de espectro de masas de astaxantina en donde se presentan tres picos principalmente; un pico correspondiente a un valor de 857.7 que se asigna a un monoéxter de astaxantina, el ácido linoleico presente para esterificar la astaxantina se observa en el pico con un valor de 579.5 y un pequeño pico con un valor de 839.7 resultante de la pérdida de agua. En general, los patrones de fragmentación de los ésteres de astaxantina fueron dominados por la pérdida de ácidos grasos y el agua (Holtin, et al., 2009; Rao, et al., 2013; Dong, et al. 2014)

Tabla 4. Compuestos separados en la cromatografía. Fuente: (Miao, et al., 2006) Contenido de esteres de astaxantina ( $\mathrm{mg} / 100 \mathrm{~g}$ de algas (con lisis)) en H. pluvialis.

\begin{tabular}{|c|c|c|}
\hline No. & Compound & Content \\
\hline 1 & Astaxanthin & 10.6 \\
\hline $5 \mathrm{a}$ & $\mathrm{ME} \mathrm{C}_{18: 4}$ & 20.4 \\
\hline $5 b$ & $\mathrm{ME} \mathrm{C}_{18: 4}$ & 8.6 \\
\hline $5 \mathrm{c}$ & $\mathrm{ME} \mathrm{C}_{18: 4}$ & 7.2 \\
\hline $6 a$ & $\mathrm{ME} \mathrm{C}_{18: 3}$ & 66.5 \\
\hline $6 \mathrm{~b}$ & $\mathrm{ME} \mathrm{C}_{18: 3}$ & 40.8 \\
\hline $7 \mathrm{a}$ & $\mathrm{ME} \mathrm{C}_{18: 2}$ & 102.5 \\
\hline $7 \mathrm{~b}$ & $\mathrm{ME} \mathrm{C}_{18: 2}$ & 8.4 \\
\hline $7 \mathrm{c}$ & $\mathrm{ME} \mathrm{C}_{18: 2}$ & 48.0 \\
\hline $8 \mathrm{a}$ & $\mathrm{ME} \mathrm{C}_{18: 1}$ & 85.4 \\
\hline $8 \mathrm{~b}$ & $\mathrm{ME} \mathrm{C}_{18: 1}$ & 77.6 \\
\hline 9 & $\mathrm{ME} \mathrm{C}_{16: 0}$ & 14.1 \\
\hline $8 \mathrm{c}$ & $\mathrm{ME} \mathrm{C}_{18: 1}$ & 36.1 \\
\hline $8 \mathrm{~d}$ & $\mathrm{ME} \mathrm{C}_{18: 1}$ & 55.1 \\
\hline 12 & $\mathrm{ME} \mathrm{C}_{16: 1}$ & 1.4 \\
\hline 13 & $\mathrm{ME} \mathrm{C}_{17: 1}$ & 3.3 \\
\hline $14 \mathrm{a}$ & $\mathrm{DE} \mathrm{C}_{18: 3} / \mathrm{C}_{18: 2}$ & 10.3 \\
\hline $14 \mathrm{~b}$ & $\mathrm{DE} \mathrm{C}_{18: 2} / \mathrm{C}_{18: 3}$ & 4.4 \\
\hline 15 & $\mathrm{DE} \mathrm{C}_{18: 2} / \mathrm{C}_{18: 1}$ & 7.9 \\
\hline 16 & $\mathrm{DE} \mathrm{C}_{18: 2} / \mathrm{C}_{16: 1}$ & 14.6 \\
\hline 17 & $\mathrm{DE} \mathrm{C}_{18: 1} / \mathrm{C}_{18: 1}$ & 12.1 \\
\hline 18 & $\mathrm{DE} \mathrm{C}_{18: 2} / \mathrm{C}_{16: 0}$ & 12.7 \\
\hline 19 & $\mathrm{DE} \mathrm{C}_{18: 0} / \mathrm{C}_{18: 1}$ & 6.3 \\
\hline $20 \mathrm{a}$ & $\mathrm{DE} \mathrm{C}_{16: 1} / \mathrm{C}_{18: 1}$ & 11.7 \\
\hline 21 & $\mathrm{DE} \mathrm{C}_{16: 0} / \mathrm{C}_{16: 0}$ & 9.9 \\
\hline $20 \mathrm{~b}$ & DE C $\mathrm{C}_{16: 1} / \mathrm{C}_{18: 1}$ & 4.6 \\
\hline 22 & $\mathrm{DE} \mathrm{C}_{18: 0} / \mathrm{C}_{18: 0}$ & 2.4 \\
\hline 23 & $\mathrm{DE} \mathrm{C}_{18: 0} / \mathrm{C}_{16: 0}$ & 2.1 \\
\hline
\end{tabular}

Note. ME, astaxanthin monoester; DE, astaxanthin diester. 
Figura. 7 Espectro de masas (APCI, el modo de iones positivos) del monoéster de astaxantina ME C18:3, junto con sus fragmentaciones principales.

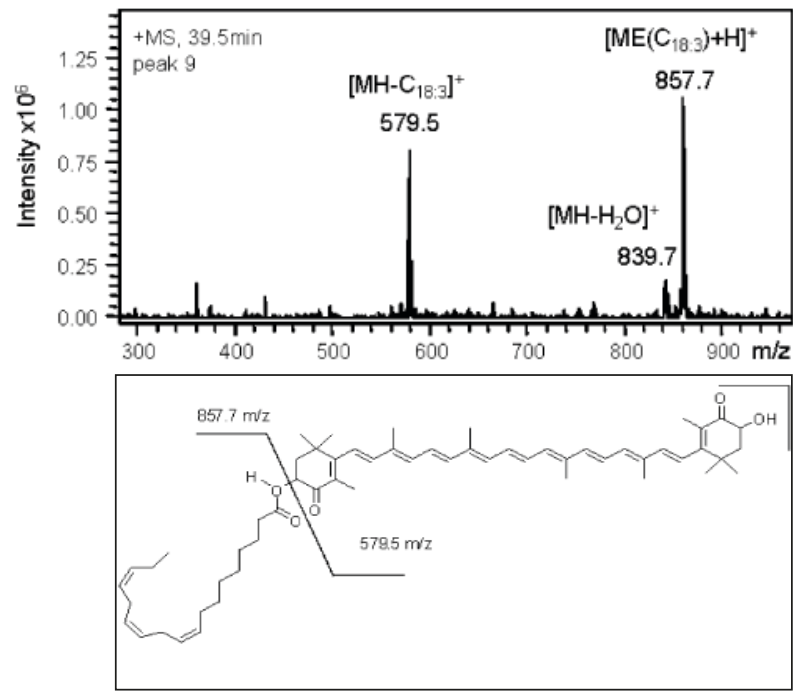

Proceso de manufactura de ésteres de astaxantina para productos alimenticios de $\boldsymbol{H}$ pluvialis con código GRAS (Generally Recognized as Safe)

El grado GRAS (generalmente reconocido y seguro) le confiere a la astaxantina unas características de seguridad en su consumo, ya que, se han consumido durante muchos años alimentos que contienen la sustancia y no se han reportado en un alto porcentaje de enfermedad o alergia. Dentro de los procesos de trasformación se hace necesaria la estabilización de la biomolécula para la comercialización y consumo.

El método de fabricación del extracto de Haematococcus, que comienza con el crecimiento de H. pluvialis células en fotobiorreactores de acero inoxidable bajo condiciones controladas. El medio de crecimiento se compone de sales minerales, oligoelementos, y un ácido de calidad alimentaria para el control de $\mathrm{pH}$. La vitamina $\mathrm{E}$ se añade a la biomasa para estabilizar la astaxantina contra la oxidación. Las paredes de las células cosechadas se trituran en agua y se secan. La fracción lipídica astaxantina que contiene éster de la biomasa seca se extrae con acetona o bien dióxido de carbono supercrítico (CO2). Los estados notificador que el proceso de fabricación es de conformidad con las buenas prácticas de fabricación actuales y los auxiliares tecnológicos utilizados son de calidad alimentaria tal como se especifica en el Food Chemical Codex. (FDA, 2010; Yin-Hu, et al., 2013; Choi, Suh, \& Lee, 2003)

Este proceso concentra la fracción lipídica incluyendo los carotenoides y es esencialmente el mismo que se usa comúnmente en la industria alimentaria para extraer la grasa de una gran variedad de productos.

\section{Estabilización}

\section{Molecular}

La astaxantina es muy inestable y sensible a la oxidación, por eso en la naturaleza se encuentra conjugada con proteínas o esterificada con ácidos grasos que le otorgan estabilidad a la molécula (Guerin, Huntley, y Olaizola, 2003). Industrialmente se han buscado formas de estabilizar la molécula. Algunos métodos son los siguientes:

1.Esterificación: Dos cadenas asimétricas unidas a los anillos bencénicos, permiten su esterificación lo que facilita su migración a través de la bicapa de las membranas celulares, incrementando su absorción por parte de los tejidos (BioNutrition, 2008).

2.Adición de vitamina E: en la industria se hace la estabilización adicionando vitamina $\mathrm{E}$ (a tocoferol).Esto se hace con el fin de que la vitamina E sea la primera en oxidarse evitando así que la astaxantina se degrade de forma más rápida.

3.Aminación o amidación: para llevar a cabo una estabilización con aminas o amidas, primero se debe realizar una esterificación y luego una aminación o amidación; su uso está enfocado principalmente en la producción de aceites para consumo animal, evitando con la aminación un enrranciamiento acelerado (De la Fuente, et al., 2002).

\section{Productos}

4. Liofilización: para una estabilización del producto una de las formas en las que se asegura la inocuidad del mismo es la liofilización, ya que, al reducir el contenido de agua se reduce el riesgo de ataque bacteriano. Adicionalmente, usar esta técnica exalta las características organolépticas sin llegar a alterar las físicoquímicas y nutricionales.

5.Secado por aspersión: dentro de este proceso se dan dos operaciones que le otorgan estabilidad a la astaxantina:

Microencapsulación y nanoencapsulación: su uso se remite a productos de alto costo y en su mayoría con fines farmacéuticos por que mejora la biodisponibilidad, otorga mayor estabilidad al producto y en el caso de la microencapsulación, genera partículas de 100 micrómetros de diámetro; para la nanoencapsulación las partículas son de $300 \mathrm{~nm}$ a 5 micras. (Bustamante, et al., 2016; Machado et al., 2014; Bustos et al., 2013; Kittikaiwan, et al., 2007)

\section{Perspectivas}

Salud: existe un gran potencial en este mercado debido a sus destacadas características antioxidantes al capturar el oxígeno 
singlete, producto de la respiración celular y producción de ATP. Esas moléculas buscaran estar apareadas con otras moléculas que se encuentren cerca, formando los radicales libres; esos radicales son neutralizados por antioxidantes producidos por el organismo o ingeridos en la dieta. La deficiencia de esos antioxidantes genera estrés oxidativo que conlleva a enfermedades degenerativas como carcinogénesis, parkinson, tumorogenesis, alzheimer, neuropatías, entre otras (BioNutrition, 2008).

Se han realizado varios estudios clínicos que muestran la efectividad de la astaxantina en la atención a enfermedades o condiciones especiales de salud; esos estudios han demostrado su efectividad al mejorar la condición de la mayoría de ellas. Se observa en la tabla un estudio clínico realizado con un total de 328 reportes de diferentes condiciones de salud en donde un $85 \%$ reportan mejoría de diferentes enfermedades presentes en distintas partes y sistemas del cuerpo humano (tabla 5).(Guerin, Huntley, \& Olaizola, 2003; Nagaraj, et al., 2012).

Tabla 5. Estudio clínico realizado por Martín Guerin (2003).

\begin{tabular}{|c|c|c|c|c|c|}
\hline \multirow{2}{*}{ Health condition } & \multirow{2}{*}{$\begin{array}{l}\text { Number of } \\
\text { reports }\end{array}$} & \multicolumn{2}{|c|}{ Improves condition } & \multicolumn{2}{|c|}{ Does not improve condition } \\
\hline & & Number & $\%$ & Number & $\%$ \\
\hline Sore muscles and joints & 146 & 128 & 8 & 18 & 12 \\
\hline Back pain & 48 & 42 & 8 & 6 & 13 \\
\hline Cholesterol & 37 & 29 & 78 & 8 & 22 \\
\hline Osteoorthritis & 20 & 19 & 95 & 1 & 5 \\
\hline Prostate & 15 & 11 & 73 & 4 & 27 \\
\hline Asthma & 13 & 11 & 85 & 2 & 15 \\
\hline Menstrual cramps & 8 & 6 & 75 & 2 & 25 \\
\hline Pheumattoid arthritits & 7 & 6 & 86 & $i$ & 14 \\
\hline Dibbetes & 5 & 1 & 20 & 4 & 80 \\
\hline Macular degeneration & 5 & 3 & 60 & 2 & \\
\hline Sunburn vegenteration & $\begin{array}{l}5 \\
5\end{array}$ & 5 & $\begin{array}{c}00 \\
100\end{array}$ & 0 & ${ }_{0}^{40}-3-3$ \\
\hline Post-surgery inflammation & 4 & 4 & 100 & 0 & 0 \\
\hline Fibromyal gia & 3 & 3 & 100 & 0 & 0 \\
\hline Gastrititis & 3 & 3 & 100 & 0 & 0 \\
\hline Gingivitis & 3 & 2 & 67 & 1 & 33 \\
\hline Peptic ucers & 2 & 2 & 100 & 0 & 0 \\
\hline Prostatititis & 2 & 2 & 100 & 0 & 0 \\
\hline Ulcerative colitis & 2 & 0 & 0 & 2 & 100 \\
\hline TOTAL & 328 & 277 & 85 & 51 & 15 \\
\hline
\end{tabular}

1.Phaffia rhodozyma: es una levadura que produce dentro de su metabolismo astaxantina en muy bajas cantidades; por lo que, se ha llevado una mutagénesis con UV o con metanosulfonato de etilo, N-metil-N'-nitro-N-nitrosoguanidina u otros análogos de bases nucleotídicas. Pasando de $650 \mu \mathrm{g} / \mathrm{g}$ de peso seco a $1.050 \mu \mathrm{g} / \mathrm{g}$ de peso seco (Aanesen, Breivik y Sanna, 2002).

2. Xanthophyllomyces dendrorhous: levadura productora de astaxantina que con agentes mutagénicos etil metano sulfonato (EMS), N-metil-N'-nitro- N-nitrosoguanidina (NTG) y radiación ultravioleta (uv) han generado mutantes superproductores de astaxantina pasando de 100 y $200 \mathrm{ppm}$ de astaxantina a 5.000 ppm o más (Aanesen, Breivik y Sanna, 2002).

\section{Conclusiones}

Para la extracción de Astaxantina se debe hace un rompimiento celular; las enzimas se presentan como una opción viable. Otra opción es tomar la Astaxantina del microorganismo Haematococcus pluvialis en su etapa vegetativa.
Para hacer una extracción con fines farmacéuticos, la mejor técnica es la de fluidos supercríticos porque logra extraer en mayor proporción y de forma más "limpia" la astaxantina, sin embargo, es necesario analizar el costo-beneficio a escala industrial.

En una cuantificación la espectrofotometría UV es una opción económica y viable.

La estabilización molecular es fundamental para hacer un aprovechamiento industrial.

Se ha demostrado con estudios clínicos que la Astaxantina tiene alta acción antioxidante por lo que su uso en medicina tiene un gran potencial. El uso de astaxantina en la producción pecuaria está ampliamente difundido y la modificación genética de levaduras y otras bacterias se convierte en una alternativa para los productores.

\section{Referencias Bibliográficas}

Aanesen, B. A., Breivik, H., y Sanna, L. I. (18 de Septiembre de 2002). España Patente n. ${ }^{\circ}$ EP20000986089.

Ahmed, F., Li, Y., Fanning, K., Netzel, M., \& Schenka, P. (2015). Effect of drying, storage temperature and air exposure on astaxanthin stability from Haematococcus pluvialis. Food Research International, 74, 231-236.

Algatechnologies Ltd. (2015). ALGA Technologies. Recuperado el Mayo de 2015, de Products: Astaxanthin: http:/www.algatech.com/products-item.asp?cat $=001$

Anarjan, N., Ping Tan, C., Arbi Nehd, I., \& Chuan Ling, T. (2012). Colloidal astaxanthin: Preparation, characterisation and bioavailability evaluation. Food Chemistry, 135, 1303-1309

BioNutrition. (Noviembre de 2008). Sp Biogroup. Obtenido de Bio Nutrition: http://www.spbiogroup.com/2010/PDFs/Functional_Ingredients/NutraXanthine_En.pdf

Bustamante, A., Masson, L., Velasco, J., del Valle, J. M., \& Robert, P. (2016). Microencapsulation of $H$. pluvialis oleoresins with different fatty acid composition: Kinetic stability of astaxanthin and alpha-tocopherol. Food Chemistry, 1013-1021.

Bustos Garza, C., Yáñez Fernández, J., \& Barragán Huerta, B. E. (2013). Thermal and pH stability of spray-dried encapsulated astaxanthin oleoresin from Haematococcus pluvialis using several encapsulation wall materials. Food Research International, 54, 641-649.

Camacho, K. J., González, M. G., \& Klotz, C. B. (Junio de 2013). Producción de Astaxantina en Haematococcus pluvialis bajo diferentes condiciones de estrés. Nova, 11(19), http:// unicolmayor.edu.co/publicaciones/index.php/nova/article/ view/227/458.

Choi, S.-L., Suh, I. S., \& Lee, C.-G. (2003). Lumostatic operation of bubble column photobioreactors for Haematococcus pluvialis cultures using a specific light uptake rate as a control parameter. Enzyme and Microbial Technology, 33, 403-409. 
Choi, Y.-E., Yun, Y.-S., Park, J. M., \& Yang, J.-W. (2011). Determination of the time transferring cells for astaxanthin production considering two-stage process of Haematococcus pluvialis cultivation. Bioresource Technology, 102, 11249-11253.

Cyanotech. (s.f.). Specification Sheet for $500 \mathrm{mg}$ Gelcaps. Recuperado el 2015, de Cyanotech: http:/www.cyanotech.com/bioastin/ specifications.html

De La Fuente, J. L.,; Peiro, E. Diez; B., Marcos, A. T., Schleissner Sánchez, C., Rodríguez Saiz, M., Barredo Fuente, J. L. (14 de Agosto de 2003). España Patente N. ${ }^{\circ} 066875$

Domínguez A, F. J. (2006). Astaxantina, el oro rojo de la microalga Haematoccocus pluvialis. Algas. Boletin Nacional de Ficología, 4-8.

Food and Drugs Administration. (6 de junio de 2010). GRAS Notice No. GRN 000294. Obtenido de FDA: http://www.fda.gov/ Food/IngredientsPackagingLabeling/GRAS/NoticeInventory/ucm 200325.htm

Fernández-Linares, L. C., Montiel-Montoya, J., Millán-Oropeza, A., \& Badillo-Corona, J. A. (septiembre-diciembre de 2012). Producción de biocombustibles a partir de microalgas. Ra Ximhai, 8(3b), 101-115.

Dong, S., Huang, Y., Zhang, R., Wang, S. and Liu, Y. (2014). Four different methods comparison for extraction of astaxanthin from green alga Haematococcus pluvialis. The Scientific World Journal, 1-7.

Gómez, P. I., Inostrozo, I., Pizarro, M., \& Jorge, P. (2013). From genetic improvementtocommercial-scalemassculture of a Chilean strain of the green microalga Haematococcus pluvialis with enhanced productivity of the red ketocarotenoid astaxanthin. AoB PLANTS 5: plt026; doi:10.1093/aobpla/plt026

Guerin, M., Huntley, M. E., \& Olaizola, M. (2003). Haematococcus astaxanthin: aplications for human health and nutrition. Trends in biotechnology, 210-216.

Harker, M., Tsavalos, A. J., y Young, A. J. (1996). Factors responsible for astaxanthin formation in the chlorophyte Haematococcus pluvialis. Bioresource Technology, 207-214.

Henriques, M., Silva, A., \& Rocha, J. (2007). Extraction and quantification of pigments from a marine microalga: a simple and reproducible method. Coimbra: Formatex.

Holtin, k., Kuehnle, M., Rehbein, J., Schuler, P., Nicholson, G., \& Albert, K. (2009). Determination of astaxanthin esters in the microalgae Haematococcus pluvialis by LC-(APCI)MS and characterization of predominant carotenoid isomers by NMR spectroscopy. Analytical and Bioanalytical Chemistry, 1613-1622.

Jaime, L., Rodríguez Meizoso, I., Cifuentes, A., Santoyo, S., Suarez, S., Ibáñez, E., \& Señorans, F. J. (2010). Pressurized liquids as an alternative process to antioxidant carotenoids' extraction from Haematococcus pluvialis microalgae. LWT-Food Science and Technology, 43, 105-112.
Japanese Conference on the Biochemistry of Lipids. (2008). LipidBank. Recuperado el 2015, de Carotenoid: Astaxanthin: http://lipidbank.jp/cgi-bin/detail.cgi?id=VCA0002

Kim, S.-K. (2015). Handbook of Marine Microalgae: Biotechlogy Advances. Elsevier Inc.

kiron, V., Phromkunthong, W., Huntley, M., Archibald, I., \& Scheemaker, G. d. (2012). Marine microalgae from biorefinery as a potential feed protein source for Atlantic salmon, common carp and whiteleg shrimp. cellana.

Kittikaiwan, P., Powthongsook, S., Pavasant, P., \& Shotipruk, A. (2007). Encapsulation of Haematococcus pluvialis using chitosan for astaxanthin stability enhancement. Carbohydrate Polymers, 70,378-385

Kobayashi, M., Kurimura, Y., Sakamoto, Y., \& Tsuji, Y. (Septiembre de 1997). Selective extraction of astaxanthin and chlorophyll from the green alga Haematococcus pluvialis. Biotechnology Techniques, 11(9), 657-660.

Lababpour, A., Shimahara, K., Hada, K., Kyoui, Y., Katsuda, T., \& Katoh, S. (2005). Fed-Batch Culture under Illumination with Blue Light Emitting Diodes (LEDs) for Astaxanthin Production by Haematococcus pluvialis. Journal of bioscience and bioengineering.

Machado Jr, F. R., Reis, D. F., Boschetto, D. L., Burkert, J. F., Ferreira, S. R., Oliveira, J. V., \& Burkert, C. A. (2014). Encapsulation of astaxanthin from Haematococcus pluvialis in PHBV by means of SEDS technique using supercritical CO2. Industrial Crops and Products, 54, 17-21.

Martínez, A. (Febrero de 2003). Universidad de Antioquia. Obtenido de Carotenoides: http://farmacia.udea.edu.co/ ff/carotenoides2001.pdf

Matamoros, V., Uggetti, E., García, J., \& Bayona, J. M. (2016). Assessment of the mechanisms involved in the removal of emergingcontaminants by microalgae from wastewater: a laboratory scalestudyVíctor. Journal of Hazardous Materials, 301, 197-205

Mendes, R. L., Nobre, B. P., Cardoso, M. T., Pereira, A. P., \& Palavra, A. F. (2003). Supercritical carbon dioxide extraction of compounds with pharmaceutical importance from microalgae. Inorganica Chimica Acta, 356, 356-328.

Miao, F., Lu, D., Li, Y., \& Zeng, M. (2006). Characterization of astaxanthin esters in Haematococcus pluvialis by liquid chromatography-atmospheric pressure chemical ionization mass spectrometry. Anal Biochemistry, 352 (2), 176-181

Nagaraj, S., M.G. Rajarama, Arulmurugan, P., Baskaraboopathy, A., Karuppasamy, K., Jayappriyan, K., Rengasamy, R. (2012). Antiproliferative potential of astaxanthin-rich alga Haematococcus pluvialis Flotow on human hepatic cancer (HepG2) cell line. Biomedicine \& Preventive Nutrition, 149-153. 
Naguib, Y. (2000). Antioxidant Activities of Astaxanthin and Related Carotenoids. Journal of Agricultural and Food Chemistry, $48,1150-1154$

Orosa, M., Franqueira, D., Cid, A., \& Abalde, J. (2005). Analysis and enhancement of astaxanthin accumulation in Haematococcus pluvialis. Bioresource Technology, 373-378.

Ramírez , D. M. (2013). Evaluación del crecimiento y producción de astaxantina por Haematococcus pluvialis en un fotobiorreactor tipo airlift. Tesis de maestría, Universidad Nacional de Colombia, Facultad de Ingeniería, Departamento de Ingeniería Química y Ambiental, Bogotá, Colombia.

Rao, A. R., Baskaran, V., Sarada, R., \& Ravishankar, G. (2013). In vivo bioavailability and antioxidant activity of carotenoids from microalgal biomass - A repeated dose study. Food Research International, 54, 711-717.

Rezanka, T., Nedbalova, L., \& Sigler, K. (2008). Identification of verylong-chain polyunsaturated fatty acids from Amphidinium carterae by atmospheric pressure chemical ionization liquid chromatography-mass spectroscopy. Phytochemistry, 69 (12) 2391-2399.

Roldán, P. L. (Febrero de 2012). Efecto del Consumo de Astaxantina en la Salud. Recuperado el Mayo de 2015, de Universitat Oberta de Catalunya: http://openaccess.uoc.edu/webapps/ o2/bitstream/10609/19841/1/Lopez 2012 O2.pdf

Seabra, L. M., \& Pedrosa, L. F. (Noviembre- Diciembre de 2010). Astaxanthin: structural and functional aspects. Revista de $\mathrm{Nu}$ trição da Puccamp, 23(6), 1041-1050.

Sila, A., Kamoun, Z., Ghlissi, Z., Makni, M., Nasri, M., Sahnoun, Z., Bougatef, A. (2015). Ability of natural astaxanthin from shrimp by-products to attenuate liver oxidative stress in diabetic rats. Pharmacological Reports, 67, 310-316.

Su, Y., Wang, J., Shi, M., Niu, X., Yu, X., Gao, L., Zhang, W. ((2014). Metabolomic and network analysis of astaxanthin-producing Haematococcus pluvialis under various stress conditions. Bioresource Technology, 170, 522-529.

Suyono, E. A., Aminin, Pradani, L., Mu'avatun, U., Habiba, R. N., Ramdaniyah, \& Rohma, E. F. (2015). Combination of blue, red, white, and ultraviolet lights for increasing carotenoids and biomass of Microalga Haematococcus pluvialis. Procedia Environmental Sciences, 28, 399 - 405.

Thana, P., Machmudah, S., Goto, M., Sasaki, M., Pavasant, P., \& Shotipruk, A. (Mayo de 2008). Response surface methodology to supercritical carbon dioxide extraction of astaxanthin from Haematococcus pluvialis. Bioresource technology, 99(8), $3110-3115$.

Tripathi, U., Sarada, R., Ramachandra Rao, S., \& Ravishankar, G. (1999). Production of astaxanthin in Haematococcus pluvialis cultured in various media. Bioresource Technology, 68, 197-199.
ValdugaI, E., TatschI, P. O., TiggemannI, L., TreichelI, H., ToniazzoI, G., ZeniI, J., JúniorII, A. F. (2009). Scielo. Obtenido de http://www.scielo.br/scielo.php?pid=S010040422009000900036\&script=sci_arttext

Vidhyavathi, R., Venkatachalam, L., Sarada, R., \& Aswathanarayana Ravishankar, G. (2007). Regulation of carotenoid biosynthetic genes expression and carotenoid accumulation in the green alga Haematococcus pluvialis under nutrient stress conditions. Journal of Experimental Botany.

Wan, M., Zhang, J., Hou, D., Fan, J., Li, Y., Huang, J., \& Wang, J. (2014). The effect of temperature on cell growth and astaxanthin accumulation of Haematococcus pluvialis during a light-dark cyclic cultivation. Bioresource Technology, 167 , 276-283.

Wenduo, Z., Wang, J., Wang, J., \& Liu, T. (2014). Attached cultivation of Haematococcus pluvialis for astaxanthin production. Bioresource Technology, 158, 329-335.

Xuebo, L., \& Toshihiko, O. (2007). Cis astaxanthin and especially 9-cis astaxanthin exhibits a higher antioxidant activity in vitro compared to the all-trans isomer. Biochemical and Biophysical Research Communications, 357(1), 187-193.

Yin-Hu, W., Jia, Y., Hong-Ying, H., \& Yin, Y. (2013). Lipid-rich microalgal biomass production and nutrient removal by Haematococcus pluvialis in domestic secondary effluent. Ecological Engineering, 60, 155-159.

You, C. J., Chul, W. C., \& Yeoung, S. Y. (2005). Combined effects of light intensity and acetate concentration on the growth of unicellular microalga Haematococcus pluvialis. Enzyme and microbial technology, 39 (3), 490-495. 